\title{
Visual short-term memory for two sequential arrays: One integrated representation or two separate representations?
}

\author{
YUHONG JIANG \\ Massachusetts Institute of Technology, Cambridge, Massachusetts \\ and \\ ARJUN KUMAR \\ Harvard University, Cambridge, Massachusetts
}

\begin{abstract}
Two dot arrays, each containing a different set of six randomly selected locations from a $5 \times 5$ matrix, were presented briefly and were separated by an interstimulus interval (ISI) of 0, 200, 500, or 1,500 msec. Subjects were asked to remember these locations and to report whether a probe dot matched the locations in memory. To find out whether the subjects had formed an integrated representation of the two arrays, the probe dot was accompanied by matrix elements from the first array, from the second array, or from both arrays. Memory for Array 1 was significantly impaired when the retrieval context was drawn from Array 2, and vice versa, suggesting that the two arrays were represented separately. This effect was observed only at an ISI of $500 \mathrm{msec}$ or longer. We propose that as Array 1 is better consolidated, its representation becomes more separated from that of Array 2.
\end{abstract}

An important challenge confronting the human visual system during natural viewing is to extract complex visual information and to retain it momentarily. A lot of vision research has focused on how the visual system perceives objects and scenes (Palmer, 1999) and how such information is retained in visual short-term memory (VSTM; Intraub, 1997; Jiang, Olson, \& Chun, 2000; Luck \& Vogel, 1997; Phillips, 1974; Rensink, O’Regan, \& Clark, 1997; Wheeler \& Treisman, 2002). Recent studies have suggested that approximately four visual objects or six spatial locations can be stored in VSTM simultaneously (Luck \& Vogel, 1997; Pashler, 1988). More features can be stored in VSTM if they conjoin to form integrated objects than if they are separate (Lee \& Chun, 2001; Luck \& Vogel, 1997; Olson \& Jiang, 2002; Wheeler \& Treisman, 2001; Xu, 2002). These studies have focused on the representation of a single visual display in VSTM. However, visual events evolve over time. Many cognitive activities, such as driving and team sports, rely on our ability to extract and retain information from multiple, successively occurring displays. In this study, we asked how two sequential arrays, separated by a variable interstimulus interval (ISI), are represented in VSTM. Specifically, are two sequential arrays represented as an integrated image or as separate images?

The present study was supported by a Helen Hay Whitney Research Fellowship. We thank Vincent Di Lollo, David Irwin, W. Trammell Neill, and Molly Potter for comments and criticisms. Correspondence should be sent to Y. Jiang, 33 Kirkland Street, Department of Psychology, Harvard University, Cambridge, MA 02138 (e-mail: yuhong@wjh.harvard.edu).
Past research on the integration of visual information has suggested that when the stimulus onset asynchrony (SOA) between two briefly presented visual arrays is within about $50 \mathrm{msec}$, the two arrays are perceived as an integrated image (Di Lollo, 1980; Phillips, 1974). This is because the leading array leaves an iconic memory (Sperling, 1960) that becomes integrated with the percept of the trailing array. However, as the SOA increases, the sensory memory of the leading array decays so that only a smaller amount of information, in a more abstract format, is retained in VSTM.

Like verbal STM, VSTM retains information about temporal sequences (Logie, 1995). For example, in a task known as the Corsi blocks, De Renzi and Nichelli (1975) pointed to a sequence of nine blocks arranged randomly on a board. Their subjects were able to recall the sequence of the blocks with some success. In another task, subjects were also able to judge whether two visual sequences, each involving a dot jumping from one location to another, were identical or different in the sequence of presentation (Logie \& Marchetti, 1991). These studies, however, have presented only a single location on each temporal frame. When information presented per array increased in complexity, such as when each array was a complex visual pattern, the subjects' recall of a series of patterns showed a dramatic recency effect, limited to the last pattern (Phillips \& Christie, 1977) or to the last three (Broadbent \& Broadbent, 1981).

An important difference between the studies on sequential retention of visual information and the more recent studies on simultaneous retention is that each temporal frame in the sequential studies contained either an 
isolated location or a single visual pattern, whereas the latter studies employed multiple separate objects or locations on a visual array. It is unclear whether we hold separate representations for successive arrays, each containing multiple separate locations, or combine them to form an integrated image.

In this article, we ask whether or not the two arrays are represented as one combined image in VSTM when the ISIs between the two arrays vary between 0 and $1,500 \mathrm{msec}$. Our approach relies on the observation that humans represent not only isolated visual objects and locations, but also the context within which the objects are presented (Biederman, 1972; Biederman, Mezzanotte, \& Rabinowitz, 1982). For example, visual search for a conjunction target is facilitated if the target is presented within an old, repeated configuration as opposed to when it is presented within new distractor locations (Chun \& Jiang, 1998). Contextual influence is revealed not only when a display was repeated many times before, but also when a display has been seen only once, as will be described below.

Retrieval from VSTM is severely disrupted by probe displays that form a configuration different from the memory display (Jiang et al., 2000). For example, suppose subjects are presented with a memory display that occupies a random set of locations-say, Locations 1-8. If the probe display also contains items at Locations 1-8 and subjects are asked whether Location 8 is one of the memory locations, performance will be quite good. In contrast, if the probe display contains items at Locations $8-15$, subjects will be close to chance to say that Location 8 is among one of the memory locations. Contextual items facilitate VSTM retrieval if they match memory encoding, and they severely disrupt VSTM retrieval if they mismatch with encoding, which conforms to the encoding specificity hypothesis (Tulving, 1974).

We rely on encoding specificity to test whether there is one integrated representation or two separate representations, employing the following reasoning. If there is one integrated representation of the combination, then a probe display containing all matrix elements presented in both arrays should match the memory representation, and a probe display containing some of the items should be less good but will still provide decent cues for memory retrieval. In contrast, if the two arrays are represented separately, a probe display containing the matrix elements presented in Array 1 should interfere with memory retrieval for a target location from Array 2, and vice versa. Thus, if the context of Array 1 interferes with the retrieval of a target in Array 2 and vice versa, there are separate representations of the two arrays. If, instead, the context of Array 1 does not interfere with the retrieval of a target in Array 2 and vice versa, the two arrays are integrated as one.

We presented subjects with two dot arrays, occupying two different sets of 6 locations in a $5 \times 5$ matrix. Each array was presented for $27 \mathrm{msec}$, and the ISI between the two arrays varied as 0, 200, or $500 \mathrm{msec}$ in Experiment $1 \mathrm{~A}$ or 1,500 msec in Experiment 1B. One second after the offset of Array 2, a probe array was presented until a response was made. The probe array contained one dot marked by a red center and several unmarked dots. The subjects were asked to remember the spatial locations occupied by the first two arrays and to decide whether the marked dot was at a previously occupied location or at a previously empty location. The marked dot could fall at a previously empty location, at one of Array 1's locations (25\%) or at one of Array 2's locations (25\%). The subjects were not explicitly instructed to combine the two arrays, nor were they encouraged to hold separate representations of the two. However, the subjects could potentially succeed in the experiment by forming a combined representation of the two arrays or by consulting each image separately. Thus, this made it possible for us to observe integration as well as separation.

We used three types of probe context. Full context was the sum of all the matrix elements presented in both arrays. Array 1 context contained matrix elements presented in the first array. Array 2 context contained matrix elements presented in the second array. Figure 1 shows a schematic sample of a trial sequence and different types of probe arrays. How would performance be affected by the mismatch between the first two arrays and the retrieval context?

\section{METHOD}

\section{Subjects}

Twenty-four students from the Boston area participated in Experiment $1 \mathrm{~A}$, and 7 students participated in Experiment 1B. Their ages ranged from 18 to 28 years. They all had normal or corrected-tonormal visual acuity and signed informed consent before the experiment. The only difference between the two experiments was the ISI.

\section{Equipment}

The subjects were individually tested in a room with dim lighting. They viewed a computer screen from an unrestricted distance of about $57 \mathrm{~cm}$, at which distance $1 \mathrm{~cm}$ corresponds to $1^{\circ}$ of visual angle.

\section{Materials}

Items (filled green circles, $0.8^{\circ} \times 0.8^{\circ}$ ) were presented on a $5 \times 5$ $\operatorname{grid}\left(9.4^{\circ} \times 9.4^{\circ}\right.$; background: midgray). Each trial started with the a fixation point for $400 \mathrm{msec}$, followed by Array 1, which filled six randomly selected cells for $27 \mathrm{msec}$. After a variable ISI $(0,200$, or $500 \mathrm{msec}$ for Experiment 1A or 1,500 msec for Experiment 1B), another array of a new set of 6 locations was presented for $27 \mathrm{msec}$. The subjects were asked to remember the locations filled by Arrays 1 and 2. After a retention interval of $1,000 \mathrm{msec}$, a probe display was presented. One of the items was the critical probe, indicated by a small red square at the center $\left(0.5^{\circ} \times 0.5^{\circ}\right)$; the rest were contextual items. The task was to report whether the cued probe marked by the red square was at one of the memorized locations (press a left key) or at a previously blank location (press a right key). The subjects were simply told to remember the 12 locations and were not explicitly asked to integrate them or to keep them separate. There were either 11 contextual items or 5 contextual items. The probe display was presented until response. Accuracy feedback, in the form of a happy or a sad face icon, immediately followed each response. One second later, the next trial commenced.

\section{Design}

The subjects were tested in three types of probe displays: full probe, Array 1 contextual probe, and Array 2 contextual probe. 


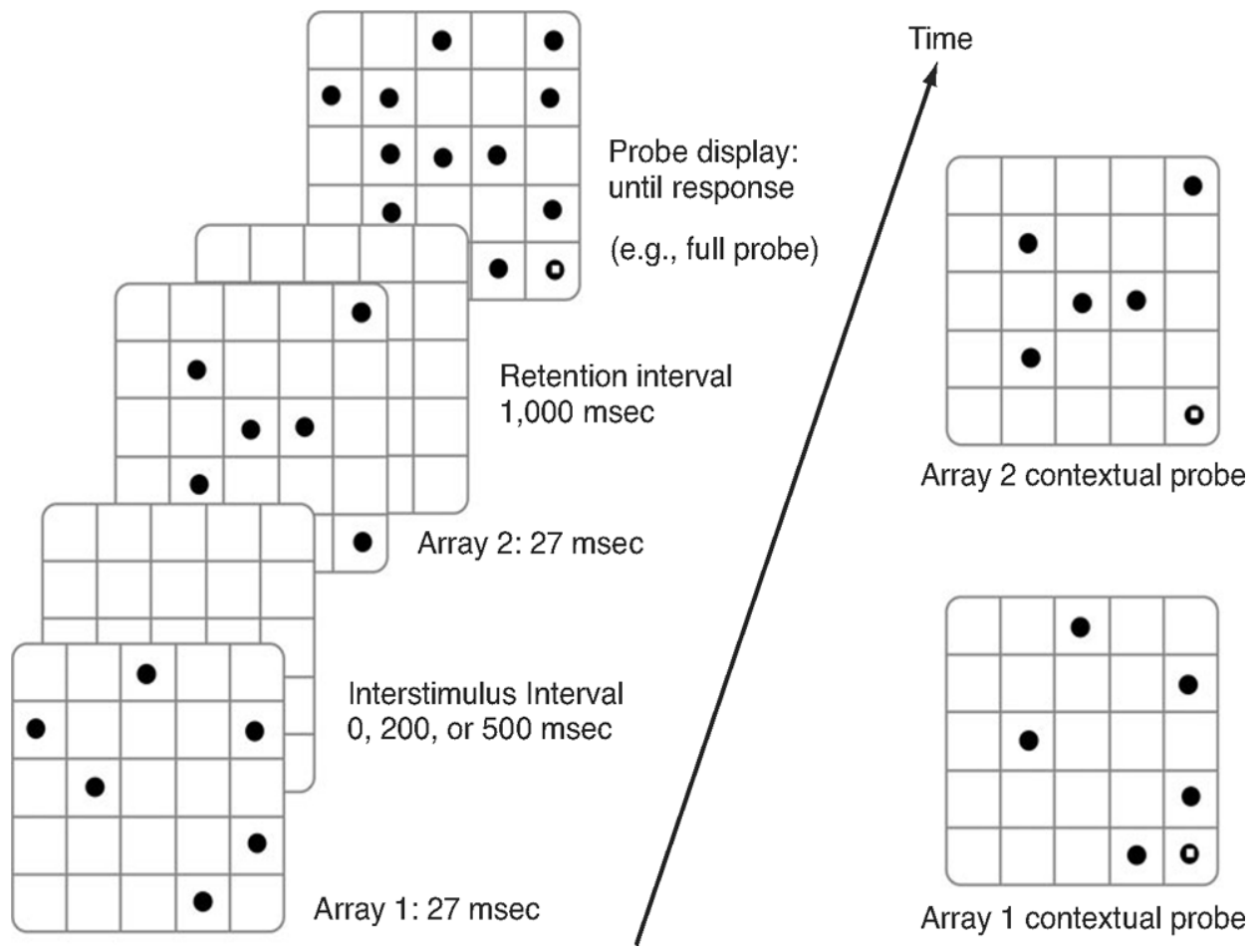

Figure 1. Schematic sample of the displays used in Experiment 1A. Left: trial sequence. Right: two types of probes (Image 1 context and Image 2 context). The critical probe is cued by a small red square inside one of the dots. The unmarked dots are contextual elements, drawn from Array 1, Array 2, or both arrays. In the experiment, half of the time memory for the first array was probed, and half of the time memory for the second array was probed.

Some subjects were also tested with mixed probes containing half of each array, and the others were tested with novel contexts containing previously unoccupied locations. Because these conditions were not diagnostic with respect to the present question, we shall not report them any further. The full probe contained all the matrix elements presented in both arrays. The Array 1 contextual probe contained matrix elements presented in Array 1. The Array 2 contextual probe contained matrix elements presented in Array 2. One third of the trials were conducted in each of the three probe conditions.

The critical probe matched Array 1 location on $25 \%$ of the trials, matched Array 2 location on $25 \%$ of the trials, and matched neither on $50 \%$ of the trials. For the latter trials, a random half was assigned to measure memory sensitivity for Array 1, and another half were assigned to measure memory sensitivity for Array 2 .

The ISI was 0, 200, or $500 \mathrm{msec}$ in Experiment $1 \mathrm{~A}$ and 1,500 $\mathrm{msec}$ in Experiment 1B.

Each subject received 6 practice trials. In Experiment 1A, 10 subjects received 288 experimental trials, and 14 received 360 experimental trials. In Experiment 1B, the subjects completed 144 experimental trials.

\section{RESULTS}

We calculated $A^{\prime}$ for each condition as a measure of the subjects' memory sensitivity (Grier, 1971). We also measured percentage correct and $d^{\prime}$. The overall pattern of results was the same whether $A^{\prime}$, percentage correct, or $d^{\prime}$ was used. $A^{\prime}$ was preferred as a measure of memory accuracy (Donaldson, 1993). Chance performance corresponds to an $A^{\prime}$ of .5, and perfect performance to 1.0.

\section{Experiment 1A: ISI $=$ 0, 200, or $500 \mathrm{msec}$}

Figure 2 shows the mean of the 24 subjects' $A^{\prime}$ values in Experiment 1A. Mean hits and false alarm rates are presented in the Appendix. In the following report, we first will describe the pattern of results at each ISI separately and then will describe a direct comparison across ISIs.

ISI $=0$ msec: Sensory integration. A multivariate analysis of variance (MANOVA) on array (whether the probe was drawn from Array 1 or Array 2) and retrieval context (whether the probe context was a full context, an Array 1 context, or an Array 2 context) revealed no significant effects for array $[F(1,23)=2.66, p>.10]$ or context $[F(2,46)=1.52, p>.20]$, and no significant interaction $[F(2,46)<1$, n.s.]. This suggests that the two arrays were represented as an integrated image and, hence, performance was independent of which array was probed and which probe context was tested. $A^{\prime}$ tended to be the highest for the full context that matched exactly with the memory, although the difference was not significant (see also Jiang et al., 2000, for a modest interference effect of a partial context).

ISI $=\mathbf{2 0 0}$ msec: Impaired consolidation of Array 1. When the ISI increased to $200 \mathrm{msec}$, a significant main effect of array was observed $[F(1,23)=19.71, p<.001]$, with superior memory for Array 2 over Array 1. The main effect of retrieval context was not significant $[F(2,46)<$ 
A. Memory for locations on Array 1

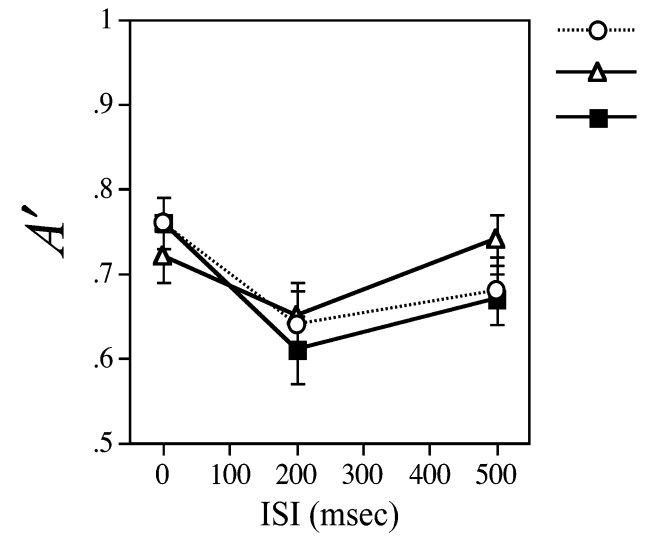

B. Memory for locations on Array 2

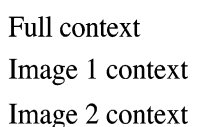

Figure 2. Results from Experiment 1A. Left: $A^{\prime}$ for Array 1 memory. Right: $A^{\prime}$ for Array 2 memory. Consolidation of Array 1 took at least $500 \mathrm{msec}$, as reflected by the increase in $A^{\prime}$ of Array 1 from 200 to 500 msec. Integration of the two arrays was perfect when the interstimulus interval (ISI) was zero, reflected by a lack of difference among different types of retrieval context. By $500 \mathrm{msec}$, the two displays were represented as separate images, revealed by a context effect (e.g., higher $A^{\prime}$ for Array 1 if the probe contained the Array 1 context rather than the Array 2 context and vice versa). The error bars show between-subjects standard errors.

$1]$, nor was the interaction significant $[F(2,46)<1]$. Memory for Array 1 was significantly above chance $(p s<$ $.01)$, but this representation did not interfere with the retrieval of Array 2, nor did the representation of Array 2 interfere with it (although there was a nonsignificant trend). This pattern is more consistent with an integrated representation than with two separate representations, although we cannot rule out the possibility that the separate representation of Array 1 might be too weak to interfere with retrieval of Array 2.

ISI $=\mathbf{5 0 0}$ msec: Separate representations of the two arrays. By an ISI of $500 \mathrm{msec}$, the consolidation of Array 1 had recovered considerably. Here, we found a significant main effect of array $[F(1,23)=17.85, p<.001]$, showing better memory for Array 2 than for Array 1 . The main effect of retrieval context was not significant $[F(2,46)<1$, n.s.], but the interaction between array and retrieval context was highly significant $[F(2,46)=9.60$, $p<.001]$, revealing an encoding specificity effect. Planned contrasts showed that when memory for Array 1 was probed, accuracy was higher if the contextual elements were from Array 1 than if they were from Array 2 $[t(23)=2.47, p<.021]$. The opposite was true when memory for Array 2 was probed, with higher accuracy for Retrieval Context 2 than for Context $1[t(23)=3.86, p<$ $.001]$. This result suggests that there were separate representations of the two arrays.

ISI effect. The above analyses show that the representation of the two arrays changed from an integrated image to separate images as the ISI increased in duration. Here, we entered array (1 vs. 2), retrieval context (full context, Array 1 context, or Array 2 context), and ISI $(0,200$, or $500 \mathrm{msec})$ in a MANOVA. A significant three-way interaction was observed $[F(4,92=2.65, p<$ $.04]$, suggesting that the mismatch in retrieval context produced different effects at different ISIs. The main effect of array was significant, revealing better memory for Array 2 than for Array $1[F(1,23)=27.31, p<.001]$. The main effect of retrieval context was not significant $[F(2,46)<1$, n.s. $]$. The main effect of ISI was significant $[F(2,46)=9.09, p<.001]$. However, ISI interacted significantly with array $[F(2,46)=4.50, p<.016]$ : Although memory for Array 1 was significantly influenced by ISI $[F(2,46)=10.40, p<.001]$, memory for Array 2 was not $[F(2,46)=1.36, p>.25]$. The other interaction effects were not significant.

\section{Experiment 1B: ISI $=1,500$ msec.}

Because combining two arrays into one integrated image may take 1,300 msec (Brockmole, Irwin, \& Wang, 2002), we tested 7 subjects, using an ISI of $1,500 \mathrm{msec}$ to find out whether the subjects would still retain separate representations at longer ISIs. Figure 3 shows the mean of the $A^{\prime}$ values. An ANOVA on array (Array 1 vs. 2 ) and context (Context 1 vs. 2 ) revealed a significant interaction $[F(1,6)=22.92, p<.003]$. Memory for Array 1 was higher when the contextual items on the display were drawn from Array 1 than when they were drawn from Array 2 $[t(6)=3.86, p<.008]$, whereas memory for Array 2 was higher when the probe context was from Array 2 than when it was from Array $1[t(6)=-3.38, p<.015]$. Thus, the subjects still maintained separate representations of the two arrays at an ISI of 1,500 msec.

\section{DISCUSSION}

In this study, we have provided strong evidence that two visual arrays were held separately in VSTM. When the SOA between the two arrays was very short (within $100 \mathrm{msec}$ or so), the two arrays were integrated at the sensory level, and VSTM for the integrated image was quite good. As the SOA increased to intermediate levels (around $200 \mathrm{msec}$ ), the first array was not successfully extracted. Its consolidation was impaired (Chun \& Pot- 


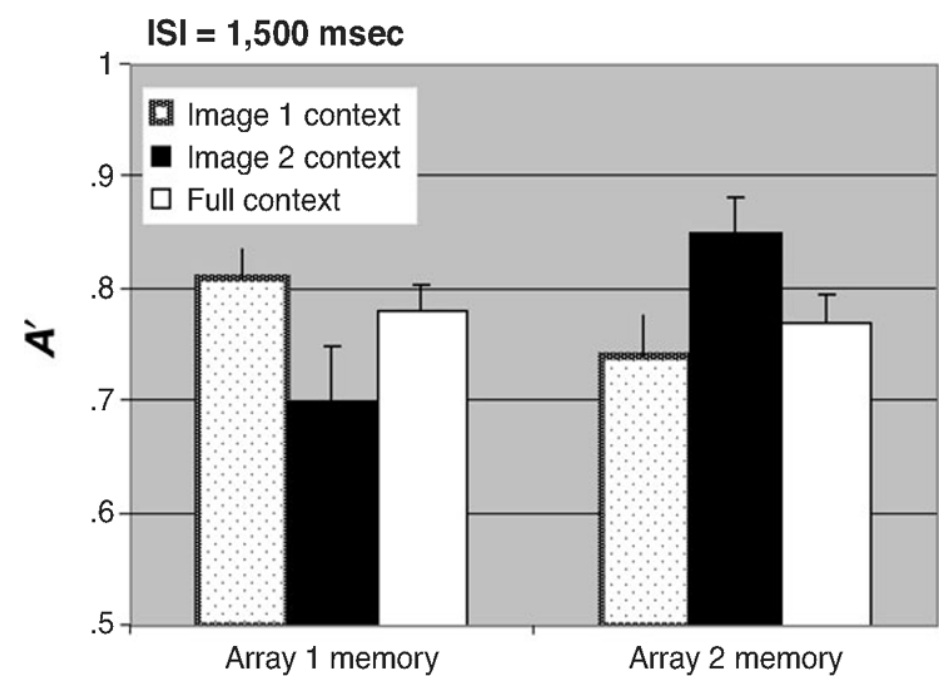

Figure 3. Results from Experiment 1B.

ter, 1995; Jiang, 2004; Potter, 1976; Raymond, Shapiro, \& Arnell, 1992). Memory for Array 2, however, was unimpaired, suggesting that the interference between the two arrays was primarily retroactive. The consolidation of Array 1 gradually improved as the SOA further increased. By $500 \mathrm{msec}$, memory for Array 1 had recovered significantly, although it still fell short of memory for Array 2 or memory for Array 1 at 0 -msec ISI $[F(1,23)=4.63, p<.042]$. As the ISI increased further to $1,500 \mathrm{msec}$, memory for Array 1 was still worse than memory for Array 2, suggesting that the second array had a competitive advantage over the first in accessing VSTM.

This study confirms that visual information is represented globally, relying on contextual elements (Chun \& Jiang, 1998; Jiang et al., 2000). Furthermore, it extends previous findings on encoding specificity to that of multiple arrays. We show here, for the first time, that it is possible to tap the representation of each array by retrieval cues that are consistent with that array. A retrieval cue that is consistent with all arrays combined facilitates memory retrieval if the arrays are represented as an integrated image, whereas a cue that is consistent with one array facilitates the retrieval of that particular array only when the arrays are represented as separate images. Our results also reveal that VSTM for complex visual arrays is not limited to the most recent presentation. This finding is more consistent with Broadbent and Broadbent's (1981) observation than with Phillips and Christie's (1977) observation on the VSTM capacity for sequential visual patterns.

In this study, we found no evidence that two visual arrays became integrated in VSTM as the first array was more successfully extracted. In a recent study, Brockmole, Wang, and Irwin (2002), however, reached an apparently different conclusion. In their study, Brockmole et al. presented subjects with 15 dots on a $4 \times 4$ grid matrix, 7 of which were presented on Array 1 and the other
8 on Array 2. The subjects were asked to detect the empty cell not occupied by either array. The authors found that performance improved gradually as the ISI increased from 100 to $500 \mathrm{msec}$, and reached asymptote at about $1,300 \mathrm{msec}$. They suggested that such a long period of ISI was necessary for subjects to form a visual image for Array 1 and to subsequently combine it with Array 2.

Although our study used a similar presentation sequence and a similar range of ISIs as Brockmole et al.'s (2002) study, there are several important methodological differences that may explain the apparent discrepancy in conclusions. First, we used the encoding specificity effect to probe the underlying representation, whereas Brockmole et al. tested integration based on subjects' ability to detect the empty cell. If subjects retained both the integrated representation and separate representations, they would show a matching effect in our paradigm (supporting separate representation) but could still perform at above-chance levels in Brockmole et al.'s paradigm (supporting integration). Thus, our data alone do not necessarily eliminate the possibility of integration. Our data does show, however, that even if the subjects did combine the two images, they did not lose the components from which the combination was derived. They further show that in our paradigm, if there was an integrated image, it was much weaker at longer ISIs than at shorter ones.

A second difference between our study and Brockmole et al.'s (2002) study is that the empty-cell detection task places a higher demand on subjects to actively combine the two arrays whereas in our task we did not place specific emphasis on integration. It is possible that subjects may rely on different strategies in the two paradigms. Informal observations suggest that in our paradigm, the subjects tried to remember all the locations from both arrays and to compare the probe with the presented locations. However in the empty-cell detection task, they tried to convert Array 1 into its negative (i.e., the un- 
occupied locations) and compare the negative of Array 1 with the dots on Array 2. Future studies should directly test how the representation of images in VSTM may be influenced by strategy and task demands (see Jiang, Kumar, \& Vickery, in press).

To summarize, as a visual image of Array 1 becomes better consolidated, it also becomes increasingly separate from the representation of a trailing array. Our study suggests that a gradual build-up of Array 1's representation might be necessary but is not sufficient for its integration with Array 2. Determining whether two consolidated images can ever be fully integrated and lose their separate representations still requires future investigation.

\section{REFERENCES}

Biederman, I. (1972). Perceiving real-world scenes. Science, 177, $77-$ 80.

Biederman, I., Mezzanotte, R. J., \& Rabinowitz, J. C. (1982). Scene perception: Detecting and judging objects undergoing relational violations. Cognitive Psychology, 14, 143-177.

Broadbent, D. E., \& Broadbent, M. H. P. (1981). Recency effects in visual memory. Quarterly Journal of Experimental Psychology, 33, $1-15$.

Brockmole, J. R., Wang, R. F., \& Irwin, D. E. (2002). Temporal integration between visual images and visual percepts. Journal of Experimental Psychology: Human Perception \& Performance, 28, 315334.

Chun, M. M., \& Jiang, Y. (1998). Contextual cuing: Implicit learning and memory of visual context guides spatial attention. Cognitive Psychology, 36, 28-71.

ChUn, M. M., \& PotTer, M. C. (1995). A two-stage model for multiple target detection in RSVP. Journal of Experimental Psychology: Human Perception \& Performance, 21, 109-127.

DE RENZI, E., \& NicheLLI, P. (1975). Verbal and non-verbal short-term memory impairment following hemispheric damage. Cortex, $\underline{11}$ 341-354.

Di LoLLo, V. (1980). Temporal integration in visual memory. Journal of Experimental Psychology: General, 109, 75-97.

Donaldson, W. (1993). Accuracy of $d^{\prime}$ and $A^{\prime}$ as estimates of sensitivity. Bulletin of the Psychonomic Society, 31, 271-274.

GrIER, J. B. (1971). Nonparametric indexes for sensitivity and bias: Computing formulas. Psychological Bulletin, 75, 424-429.

InTRaUB, H. (1997). The representation of visual scenes. Trends in Cognitive Sciences, 1, 217-222.

JIANG, Y. (2004). Time window from visual images to visual short-term memory: Consolidation or integration? Experimental Psychology, 51, 45-51.

JiAnG, Y., Kumar, A., \& Vickery, T. J. (in press). Integrating visual arrays in visual short-term memory. Experimental Psychology.

JiAnG, Y., Olson, I. R., \& Chun, M. M. (2000). Organization of visual short-term memory. Journal of Experimental Psychology: Learning, Memory, \& Cognition, 26, 683-702.

LEE, D., \& CHUN, M. M. (2001). What are the units of visual short-term memory, objects or spatial locations? Perception \& Psychophysics, 63, 253-257.

LoGIE, R. H. (1995). Visuospatial working memory. East Sussex, U.K.: Erlbaum.

Logie, R. H., \& MARChetTI, C. (1991). Visuospatial working memory: Visual, spatial or central executive? In R. H. Logie \& M. Denis (Eds.), Mental images in human cognition (pp. 105-115). Amsterdam: North-Holland.

LucK, S. J., \& Vogel, E. K. (1997). The capacity of visual working memory for features and conjunctions. Nature, 309, 279-281.

OLSON, I. R., \& JIANG, Y. (2002). Is visual short-term memory object based? Rejection of the "strong-object" hypothesis. Perception \& Psychophysics, 64, 1055-1067.

Palmer, S. E. (1999). Vision science. Cambridge, MA: MIT Press.

PAshler, H. (1988). Familiarity and visual change detection. Perception \& Psychophysics, 44, 369-378.

PHILLIPS, W. A. (1974). On the distinction between sensory storage and short-term visual memory. Perception \& Psychophysics, 16, 283-290.

Phillips, W. A., \& Christie, D. F. M. (1977). Interference with visualization. Quarterly Journal of Experimental Psychology, 29, 637 650 .

Potter, M. C. (1976). Short-term conceptual memory for pictures. Journal of Experimental Psychology: Human Learning \& Memory, 2,509-522.

RAYMOND, J. E., Shapiro, K. L., \& ARnell, K. M. (1992). Temporary suppression of visual processing in an RSVP task: An attentional blink? Journal of Experimental Psychology: Human Perception \& Performance, 18, 849-860.

Rensink, R. A., O'Regan, J. K., \& Clark, J. J. (1997). To see or not to see: The need for attention to perceive changes in scenes. Psychological Science, 8, 368-373.

SpERLING, G. (1960). The information available in brief visual presentations. Psychological Monographs: General \& Applied, 74, 1-29.

Tulving, E. (1974). Cue-dependent forgetting. American Scientist, 62, 74-82.

Wheeler, M. E., \& Treisman, A. M. (2002). Binding in short-term visual memory. Journal of Experimental Psychology: General, $\mathbf{1 3 1}_{2}$ 48-64.

XU, Y. (2002). Limitations of object-based feature encoding in visual short-term memory. Journal of Experimental Psychology: Human Perception \& Performance, 28, 458-468.

\section{Appendix}

Hit and False Alarm Rates Observed for Each Interstimulus Interval (in Milliseconds) in Experiments 1A and 1B

\begin{tabular}{|c|c|c|c|c|c|c|c|c|c|c|c|c|c|c|c|c|}
\hline \multirow[b]{3}{*}{ Memory Array } & \multirow[b]{3}{*}{ Probe } & \multicolumn{8}{|c|}{ Hit Rate (\%) } & \multicolumn{7}{|c|}{ False Alarm Rate (\%) } \\
\hline & & \multicolumn{2}{|c|}{0} & \multicolumn{2}{|c|}{200} & \multicolumn{2}{|c|}{500} & \multicolumn{2}{|c|}{$1,500^{*}$} & \multicolumn{2}{|c|}{0} & \multicolumn{2}{|c|}{200} & \multicolumn{2}{|c|}{500} & $1,500 *$ \\
\hline & & $M$ & $S E$ & $M$ & $S E$ & $M$ & $S E$ & $M$ & $S E$ & $M$ & $S E$ & $M$ & $S E$ & $M$ & $S E$ & $M S E$ \\
\hline \multirow{3}{*}{ Image 1 memory } & Context 1 & 56 & 4 & 62 & 4 & 6 & 3 & 64 & 5 & 27 & 4 & 40 & 4 & 33 & 4 & 24 \\
\hline & Con & 61 & 4 & 58 & - & 5 & 4 & 50 & 8 & 0 & 5 & 44 & 4 & 35 & 3 & 25 \\
\hline & Full probe & 59 & 4 & 67 & 5 & 68 & 3 & 68 & 4 & 22 & 4 & 49 & 5 & 43 & 4 & 31 \\
\hline \multirow[t]{3}{*}{ Image 2 memory } & Cont & 64 & 3 & 66 & 4 & 60 & 4 & 66 & 7 & 25 & 4 & 27 & 3 & 25 & 4 & 30 \\
\hline & Cont & 60 & 4 & 56 & 5 & 63 & 4 & 60 & 6 & 20 & 4 & 18 & 4 & 11 & 2 & 12 \\
\hline & Full probe & 59 & 5 & 66 & 3 & 70 & 4 & 60 & 4 & 14 & 4 & 27 & 4 & 24 & 4 & 20 \\
\hline
\end{tabular}

*Data from Experiment 1B. 